\section{Transplantation Antigens in Rhesus Monkeys}

WE read your interesting editorial ${ }^{1}$ dealing with our work on the major transplantation antigens of rhesus monkeys, the RhL-A system. Your communication ends: "The previously held concept of $\mathrm{H}-2$ genetic structure is now very much open to doubt. If further work shows that the RhL-A system also consists of two segregant series of antigens it is bound to undermine even more the concept of $\mathbf{H}-2$ as a system of multiple, pseudoallelic antigens".

It might be worth mentioning that in the months that passed since we submitted our communication, experimental evidence has been obtained which strongly suggests that there are indeed two segregant series of alleles which determine the antigens of the RhL-A system. Further, our work on chimpanzee cells revealed that the principal leucocyte antigens of that primate species may also be governed by one genetic system, tentatively called ChL- ${ }^{3}$. Recent evidence obtained in cross-species typing between chimpanzee and man indicated that some of these specificities are closely related to human HL-A antigens of the first or "LA" series of alleles, while several other chimpanzee antigens resemble specificities of the second or "Four" series of HL-A (this last group of chimpanzee specificities exhibited a phenotypic distribution in 200 animals, suggesting their determination by a series of allelic genes; the others did not fit into the same allelic series). The data therefore suggest that the tissue antigens of chimpanzees may also be determined by two series of closely linked genes ${ }^{3}$.

The opinion expressed in your editorial that, as in the human HL-A system, two linked series of allelic genes may determine the major histocompatibility antigens also in other mammalian species seems to be corroborated by our preliminary data for monkeys and chimpanzees.

\section{H. BALNER}

J. J. VAN ROOD

Radiobiological Institute TNO, Rijswijk $\mathbf{Z H}$

Received April 30, 1971.

1 Nature, 230, 279 (1971).

2 Balner, H., Gabb, B. W., Dersjant, H., Van Vreeswijk, W., and van Rood, J. J., Nature New Biology, 230, 177 (1971).

${ }^{3}$ Balner, H., et al., Transplantation, 11, 309 (1971).

${ }^{4}$ Balner, H., et al., Transplant. Proc. (in the press).

\section{Communication between Cells of Different Type}

MANY types of cells have membrane junctions so organized as to allow the passage of molecules from the interior of one cell to another with little loss to the outside ${ }^{1-6}$. At least in some cases, a large part if not all of the surface membrane seems to be capable of this organization, for if the junction is broken and the cell pair rejoined at some other place, the system can become fully communicating within seconds or minutes ${ }^{7,8}$. Evidently the membranes must undergo profound structural changes in such a way that previously impermeable surface regions become highly permeable. This implies a close spatial matching of the joining regions on the two membranes. The question arises whether such matching can be effected between membranes that differ from each other. To find something out about this problem, we have paired cultured cells from different organs and species, measuring the communication between cells electrically.

We have used rabbit lens epithelial cells (lens) ${ }^{9}$, 3T3 mouse fibroblasts transformed by simian virus 40 (SV40-3T3) ${ }^{10}$, hamster kidney fibroblasts $(\mathrm{BHK}-21)^{\mathbf{1 1}}$, a polyoma-transformed derivative of BHK-21 (P $\gamma \mathrm{Y})$, a mutant of $\mathrm{P} \gamma \mathrm{Y}, \mathrm{P} \gamma \mathrm{Y}-$
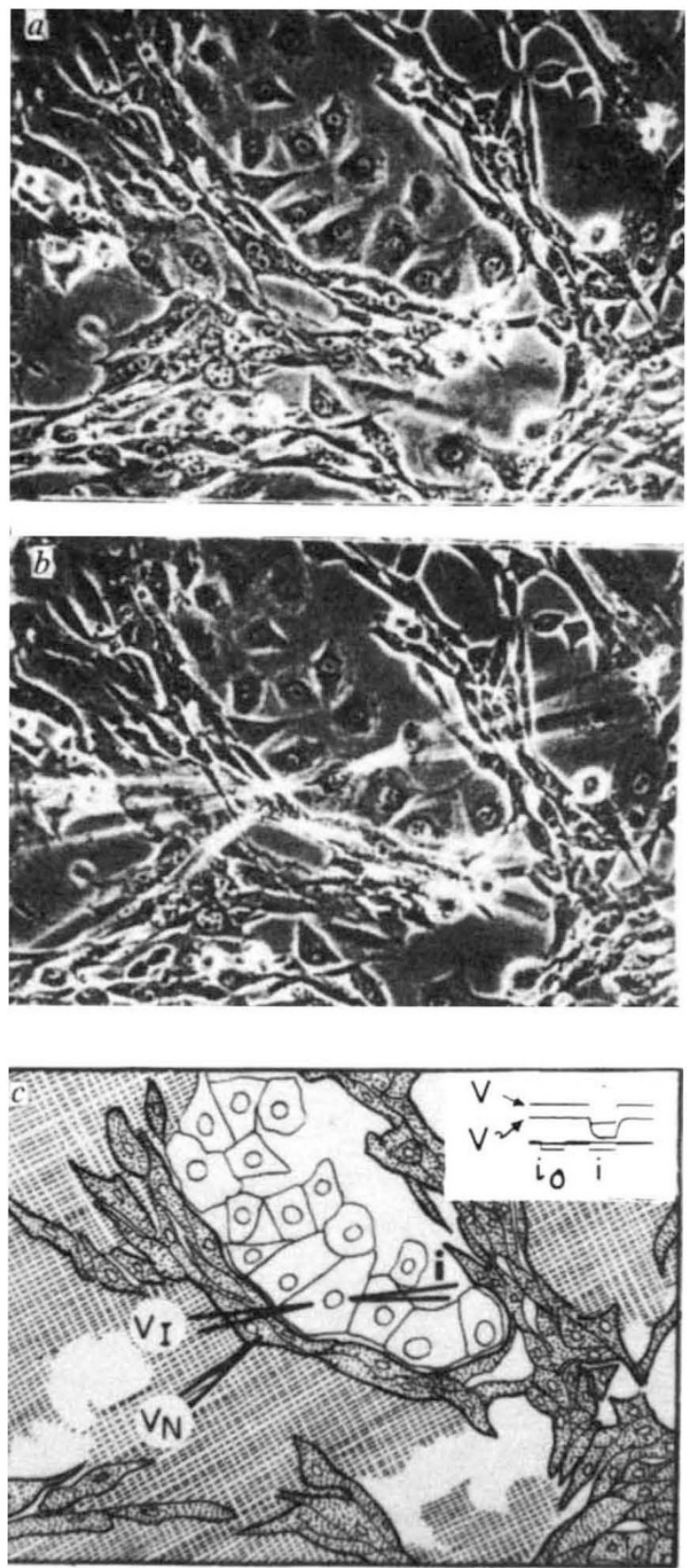

Fig, $1 a$ A co-culture of hamster kidney fibroblasts (BHK-21) and rat liver cells, shown in $b$ with 3 microelectrodes in intracellular position for measurement of coupling. On the tracing below, the BHK cells are dotted and the liver cells clear. A current-delivering $(i)$ and voltage-recording $\left(V_{1}\right)$ electrode are inside a liver cell and a second voltage-recording electrode V is in a BHK cell. Inset, an oscilloscope sample record from a measurement on a similar co-culture. (The first current pulse $\left(i_{0}\right)$ is delivered with a fourth electrode outside the cells.) $i=$ $1 \times 10^{-8} \mathrm{~A} ; 100 \mathrm{~ms} ; \mathrm{V}_{1}=25 \mathrm{mV}$.

TG-TGR (TGR) ${ }^{12}$ and rat liver epithelial cells (liver) ${ }^{13}$. The first two cell types were grown in Eagle's medium ${ }^{14}$ as modified by Vogt and Dulbecco ${ }^{15}$ (ED medium) with $15 \%$ calf serum, the next two in BHK-21 medium ${ }^{11}$ with $10 \%$ calf serum and $10 \%$ tryptose phosphate broth, and the last one in ED medium with $10 \%$ foetal calf serum. All cell mixtures were grown in ED medium with $15 \%$ calf serum. The cell cultures were kept in plastic Petri dishes at $37^{\circ} \mathrm{C}$ and equilibrated at $p \mathrm{H} 7.3$ with a moist $\mathrm{CO}_{2}$-air mixture.

Intercellular communication was measured by injecting an electrical current $(i)$ into one cell (1) of a group of cells in visible contact with each other and measuring the resulting voltages in this cell $\left(V_{1}\right)$ and another cell $\left(V_{\mathrm{N}}\right)$ of the group, contiguous with cell 1 or separated by one or two inter- 